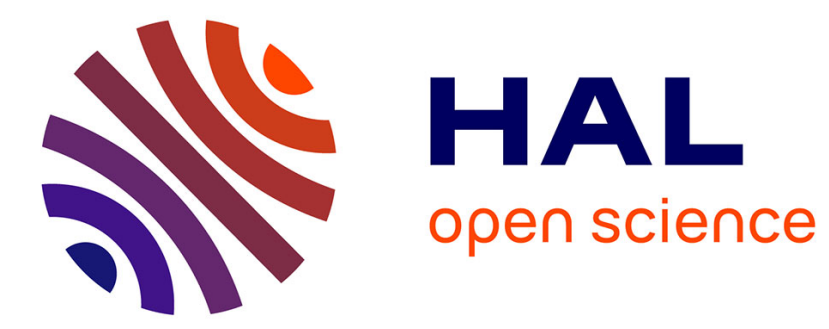

\title{
Mill, Darwin et l'argument du dessein
}

Denis Forest

\section{To cite this version:}

Denis Forest. Mill, Darwin et l'argument du dessein. Cahiers philosophiques, 2017, 148, pp.9-25. 10.3917/caph.148.0009 . hal-03237991

\section{HAL Id: hal-03237991 \\ https://hal.science/hal-03237991}

Submitted on 26 May 2021

HAL is a multi-disciplinary open access archive for the deposit and dissemination of scientific research documents, whether they are published or not. The documents may come from teaching and research institutions in France or abroad, or from public or private research centers.
L'archive ouverte pluridisciplinaire HAL, est destinée au dépôt et à la diffusion de documents scientifiques de niveau recherche, publiés ou non, émanant des établissements d'enseignement et de recherche français ou étrangers, des laboratoires publics ou privés. 
MILL, DARWIN

ET L'ARGUMENT DU DESSEIN ${ }^{1}$

Stuart Mill examine dans le dernier des Trois Essais sur la religion l'argument du Dessein. Ce faisant, il l'évalue selon les normes épistémiques définies dans la Logique tout comme il examine en parallèle l'hypothèse présentée par Darwin dans l'Origine des Espèces qui se propose d'expliquer les mêmes phénomènes. Pour comprendre le jugement nuancé que porte Mill sur l'argument du Dessein, et la manière dont l'alternative darwinienne ne lui paraît pas pouvoir l'emporter sur le Dessein au moment où il écrit, il faut faire intervenir trois niveaux d'analyse : la relation entre méthode inductive et méthode hypothétique (théorie de la connaissance), le choix du phénoménalisme qui limite l'engagement naturaliste (métaphysique), la question de l'espoir raisonnable (philosophie morale). La philosophie de la religion, à travers la question du Dessein et la solution sceptique qui lui est proposée, engage ainsi l'unité de la philosophie de Mill.

\section{Introduction : des réticences inattendues}

On ne peut pas découvrir le traitement que Stuart Mill réserve à l'argument du Dessein dans les Essais sur la religion sans une certaine surprise ${ }^{2}$. Pour le lecteur d'aujourd'hui, prompt à réfléchir en termes de tradition de pensée, Mill peut être situé dans la filiation de Hume et Hume lui-même est connu pour la critique incisive de l'argument du Dessein contenue dans les Dialogues sur la religion naturelle où Philon pointe la faiblesse du raisonnement inductif qui paraît si

\footnotetext{
${ }^{1}$ Je remercie Jean Gayon et Vincent Guillin pour leurs suggestions.

2 Toutes les références à Mill seront à l'édition des Collected works, avec chiffre romain pour le volume, et chiffre arabe pour la page. Les Trois Essais sur la Religion édités pour la première fois à titre posthume en 1874 regroupent " Nature ", "Utilité de la religion » et " Théisme ». " Nature » traite de la vacuité du commandement " suivre la nature ", et rejette l'idée de l'omnipotence divine. "Utilité de la religion » traite en particulier des relations entre religion et moralité. Le troisième, "Théisme " est celui qui examine directement la vérité de la religion et les preuves de Dieu, dont l'argument du Dessein (Mill, X, p. 446-450). Le troisième Essai, dont on estime qu'il a été écrit entre 1868 et 1870 (Raeder, 2002, p. 167), est nettement postérieur aux deux autres.
} 
probant à Cléanthe et aux amis de la théologie rationnelle 3 . Dans "Théisme " le dernier Essai majeur qu'il devait rédiger, Mill examine pourtant l'argument du Dessein comme un argument qui mérite examen, dans un contexte où il rejette d'autres arguments rationnels en faveur de l'existence d'un créateur. Si l'existence du monde n'exige pas de poser un créateur transcendant qui en rendrait raison et ferait office de première cause, pour Mill la configuration du monde demande, elle, une explication, et en particulier la structure des organismes, explication que l'argument du Dessein prétend fournir. Certes, on se souvient des mises en garde contre toute précipitation lorsqu'on veut à tout prix poser une tradition empiriste ${ }^{4}$ : Mill, qui ne se réfère guère à $\mathrm{Hume}^{5}$, a recours exceptionnellement au terme "empirisme", et l'utilise en un sens péjoratif au début du Livre VI de la Logique ${ }^{6}$. Mais un autre aspect du texte ne peut qu'augmenter notre perplexité : lorsque Hume affirmait que l'inférence de la convenance mutuelle entre structures et fonctions des parties des organismes à une conception intelligente des êtres vivants témoignait seulement d'une partialité en notre faveur (la pensée est un des principes actifs dans l'univers, pourquoi serait-elle le principe de toute chose), il affaiblissait l'argument du Dessein dans un contexte où aucune explication autre que téléologique n'était disponible. Mill de son côté, écrit l'essai "Théisme " après la publication de l'Origine des espèces et il ne manque pas de rappeler que la conception de la vie en termes de "survie des plus aptes" offre une voie alternative au Dessein". Comment comprendre alors la conclusion à laquelle il aboutit, où en termes de probabilité, la balance penche en faveur du Dessein, et non de l'action de la sélection naturelle ? Cette question, qui sera celle du présent article, mérite d'autant plus d'être posée que Mill accepte pourtant dans les mêmes Essais l'idée d'une forme d'émergence diachronique où l'intelligence apparaît

\footnotetext{
${ }^{3}$ Philon semble se rétracter entièrement plus loin dans les Dialogues (XIlème partie, début), mais cette rétractation est suspecte et ce qui a marqué l'histoire est surtout la critique de l'argument du Dessein telle qu'elle apparaît dans la seconde partie.

${ }^{4}$ Van Fraassen, 2002. P. 33.

${ }^{5}$ L'unique mention du nom de Hume dans l'Essai sur le Théisme (X, p. 470) concerne sa position sur les miracles, telle qu'elle est exposée dans l'Enquête, et non les Dialogues.

${ }^{6}$ Mill, VIII, p. 834.

${ }^{7}$ Mill, X, p. 449.
} 
tardivement dans la nature au lieu d'être placée au commencement de toute chose car rien d'inférieur à elle ne pourrait la produire ${ }^{8}$.

Un autre motif d'interrogation est la comparaison entre l'attitude de Whewell et celle de Mill vis-à-vis de Darwin. Whewell, l'autre grande figure de la philosophie des sciences dans l'Angleterre victorienne avec Herschel et Mill, dont on sait en particulier depuis le travail de Michael Ruse l'importance qu'il a eu aux yeux de Darwin lui-même pour l'idée que celui-ci se faisait de la confirmation d'une théorie $^{9}$, a affirmé ses positions anti-évolutionnistes à plusieurs reprises. II exprime ces positions dans son Bridgewater Treatise de 1833, intitulé Astronomy and general physics, considered with Reference to Natural Theology, où il s'inscrit comme l'a rappelé David Hull ${ }^{10}$, dans la tradition de Richard Bentley dans le projet d'une conciliation entre science et théologie. II exprime également ces positions en colligeant des textes de son History of the inductive sciences dans Indications of the creator (1845), un volume publié en réponse aux Vestiges of the natural history of creation de Robert Chambers (1844). Dans sa Préface à la première édition de ce texte, Whewell notait qu'un avantage des considérations qu'il présentait était de ne pas avoir été à l'origine écrites dans une perspective théologique ${ }^{11}$ : seule une science indépendante de toute religion et de toute apologétique peut apporter quelque chose à la cause de la théologie naturelle. Dans L'History, en examinant la question de savoir si " des êtres organisés peuvent, par le seul agencement de causes naturelles, passer du type d'une espèce à celui d'une autre ${ }^{12}$, Whewell, s'appuyant sur l'autorité de Cuvier, prend parti en faveur d'une extension limitée des modifications possibles des êtres vivants, et contre la transmutation des espèces, laquelle imposerait "d'abandonner la croyance en une adaptation de la structure de chaque créature au mode d'être auquel elle est destinée ${ }^{13}$. Cependant, Whewell se refuse à élaborer une critique de l'Origine des espèces ${ }^{14}$ et il se contente en 1864

\footnotetext{
${ }^{8}$ Mill,, X, p. 440.

${ }^{9}$ Ruse, 1975.

${ }^{10}$ Hull, 1973.

${ }^{11}$ Whewell, 1846, p. 32.

${ }^{12} \mathrm{Ibid}$, p. 98.

${ }^{13}$ Ibid., p. 99.

${ }^{14}$ Lettre de Whewell au révérend D. Brown d'Aberdeen, 26 octobre 1863, citée par Snyder, 2006, p. 196, note 135.
} 
de faire réimprimer le Traité de 1833 sans en altérer le contenu, en l'augmentant d'une nouvelle Préface qui réaffirme la connexion nécessaire entre adaptation et Dessein.

On peut s'étonner, étant donné le long débat qui a opposé Mill à Whewell sur la vraie théorie de l'induction et sur la méthodologie des sciences de la nature ${ }^{15}$, que le premier ne saisisse pas l'occasion qui lui est donnée de se démarquer une nouvelle fois du second. Selon Whewell, l'esprit antireligieux de certains scientifiques, comme Laplace ${ }^{16}$, et leur rejet explicite des causes finales, est lié au type de science qu'ils pratiquent et aux conséquences de la division du travail à l'intérieur du monde savant ${ }^{17}$. Pour lui, en effet, la démarche de subsomption propre à l'induction du découvreur prépare l'esprit d'un savant comme Newton à embrasser une perspective toujours plus large, à rechercher un principe explicatif toujours plus élevé, à postuler ce qui ne se rencontre pas directement dans l'expérience ordinaire; en revanche, la démarche déductive de ceux qui tirent des principes premiers leurs conséquences renforce une tournure d'esprit opposée, prompte à rejeter ce qui est extra-ordinaire. Selon Whewell, il est faux que la science réfute la religion, mais il est vrai qu'une certaine pratique de la science prédispose à une forme de cécité vis-à-vis du Dessein. Et réfléchir sur la démarche de la science, $c^{\prime}$ est s'expliquer pourquoi Laplace manque le Dessein comme s'expliquer que Newton ait été capable de le reconnaître. Lorsqu'il aborde le Dessein, Mill, si opposé à Whewell sur de nombreux points, si soucieux de travailler à rendre possible un monde intellectuel moins conservateur ${ }^{18}$, en vient à minorer Darwin et il ne saisit pas l'occasion de s'opposer frontalement à I'alliance du théisme et de la science telle que Whewell la défend. Les trois Essais sur la Religion aboutissent au scepticisme, non à l'athéisme. Mill rejette une certaine conception de la divinité, comme il l'avait déjà fait lors de sa polémique avec Mansel au septième chapitre d'Un examen de la philosophie de sir William Hamilton $^{19}$, mais il ne réprouve pas toute référence du divin. Reconstruire la

\footnotetext{
15 Snyder, 2006, chapitres I à III.

${ }^{16}$ Sur Laplace, voir Merleau-Ponty, 1983.

17 Astronomy and general physics, considered with Reference to Natural Theology, Bridgewater Treatise, Partie III, chapitre VI : "Deductive habits".
}

18 Snyder, 2006.

${ }^{19}$ Mill, IX, p. 103. 
position de Mill sur le Dessein, c'est à la fois préciser à quoi correspond un tel scepticisme, ce qui le motive, et comment il engage l'unité peu apparente, mais bien réelle, de la philosophie de Mill, ainsi que des questions sur la relation entre science et philosophie qui sont toujours les nôtres.

\section{Mill sur le Dessein : vertus et limites de la méthode de concordance}

Dans sa revue des arguments en faveur de l'existence de la Divinité, Mill aborde en dernier l'argument " à partir des marques du Dessein dans la Nature ${ }^{20}$, un argument qui " enfin, est doué d'un caractère authentiquement scientifique ". Cependant, la question préalable est de préciser quelle en est la formulation correcte. Une manière canonique de le présenter est la suivante: 1 . nous savons d'expérience que les objets qui sont fabriqués par un concepteur intelligent en vue d'une fin ont des propriétés de type P. 2. Or certaines choses naturelles présentent de telles propriétés. 3. "Nous sommes fondés, à partir de cette grande similitude des effets, à inférer une similarité dans la cause, et de croire que les choses qui dépassent le pouvoir de fabrication de l'homme, mais qui ressemblent aux œuvres de l'homme en tout sauf en puissance [requise pour les exécuter] doivent avoir été faites par une Intelligence armée d'un pouvoir plus grand que le pouvoir humain ".

Cette formulation classique rappelle l'exemple célèbre de la montre que William Paley avait présenté, mais elle ne paraît cependant pas à Mill présenter l'argument du Dessein sous son meilleur jour. Car elle infère une similitude des causes à partir d'une similitude des effets en affirmant qu'il y a ressemblance entre les choses naturelles et les produits de l'intelligence humaine, sans souligner quelle est la nature de cette ressemblance qui exige le recours à une intelligence fabricatrice. Dans l'exemple de la montre sur le rivage, nous associons sa présence à une présence humaine, mais selon Mill nous aurions pu en faire autant avec une trace de pas ou quelque relique insignifiante ${ }^{21}$ : et de même, la présence de coprolithes dans des couches de l'écorce terrestre permet aux géologues d'inférer la présence d'animaux dans des périodes reculées. Ces exemples montrent que nous pouvons inférer l'origine d'une trace à partir de celle-ci dans des cas où la trace n'est l'effet d'aucun dessein. Des créatures intelligentes peuvent laisser derrière elles des vestiges sans que ces vestiges portent sur eux la marque de leur intelligence. Si ce n'est pas seulement l'origine

\footnotetext{
${ }^{20}$ Mill, X, p. 446.

${ }^{21}$ Ibid, p. 447.
} 
transcendante des êtres vivants, mais la conception des êtres vivants par une intelligence qui est en jeu, alors il faut présenter l'argument d'une autre manière de manière à faire ressortir sa force, celle d'un véritable argument inductif.

En examinant pour des raisons de commodité, le cas particulier de l'œil plutôt que l'argument dans sa généralité, la question est alors de savoir quel est le type d'argument inductif auquel correspond l'argument du Dessein, et quelle en est la force. Parmi les quatre méthodes distinguées au chapitre VIII du Livre III de la Logique (concordance, différence, résidus et variation concomitante), c'est la première, la Méthode de concordance, qui est suivie par l'argument du Dessein. Chaque méthode entend « identifier, parmi les circonstances qui précèdent ou suivent un phénomène, celles avec lesquelles il est réellement relié par une loi invariable ${ }^{22}$. La méthode de concordance effectue cette recherche comparant des cas et en identifiant quelle circonstance précède immuablement le phénomène considéré, quel que soit le contexte où il apparaît : si $A B C$ est suivi de $a b c$ et que ADE est suivi de ade, A est identifié comme l'antécédent invariable du phénomène $a$, du moins si on admet qu'un effet doit avoir un antécédent causal nécessaire ${ }^{23}$. La méthode s'applique selon Mill à la question du Dessein en ceci que 1. Les parties de l'œil ont en commun une seule propriété distinctive (c'est-à-dire non partagée avec d'autres segments de l'organisme): contribuer à la vision. 2. Commençant dans le temps, ces parties doivent résulter d'une cause ou d'un complexe de causes qui leur est commun. 3. Le fait pour les toutes parties de l'œil de " conspirer » à la production de la vision, et la nature de la cause qui a produit ces parties et qui les a dotées, elles seules, de cette propriété, doivent être reliés. 4. Le nombre de cas convergents dans la nature élimine l'hypothèse selon laquelle la possession commune de la même propriété par chaque partie de l'œil est l'effet du hasard.

Si on transpose les conventions de la Logique (des minuscules pour les effets, des majuscules pour les causes), $a b c$ (la cataracte) et ade (la rétine) doivent avoir un antécédent commun $\mathrm{A}$ qui explique la récurrence de $a$, c'est-à-dire la récurrence de la propriété " qui contribue à la vision ». On voit que l'application de la méthode de concordance est hétérodoxe : il ne s'agit pas de détecter un antécédent invariable dans diverses occurrences du même phénomène, mais un antécédent invariable pour plusieurs phénomènes qui instancient la même propriété. Mill conclut alors : « La vision, étant un fait qui suit et qui ne précède

\footnotetext{
${ }^{22}$ Mill, VII, p. 388.

${ }^{23}$ Skorupski, 1989, p. 185.
} 
pas la réunion des parties organiques de l'œil, peut seulement être connectée avec la production de cette structure en ayant le caractère d'une cause finale, et non efficiente: c'est-à-dire que ce n'est pas la vue elle-même mais l'idée antécédente de la vue, qui doit être cause efficiente. Mais ceci caractérise aussitôt l'origine comme procédant d'une volonté intelligente ${ }^{24}$.

C'est à ce point qu'intervient dans "Théisme " la référence à Darwin, dont on sait qu'il a retenu ce même exemple de l'œil au chapitre VI de l'Origine des espèces. La référence à Darwin va de pair avec une décomposition de l'argument du Dessein. Pour Mill il y a en effet deux moments dans le raisonnement inductif tel qu'il l'a reconstruit. Le premier moment établit qu'il doit exister une connexion entre d'une part la récurrence d'une propriété que partagent des effets, et d'autre part la nature de la cause que ces effets ont en commun. Le second moment consiste à spécifier la nature de cette cause, en faisant appel à une forme de causalité par intention. Mill précise alors que "cette seconde moitié de l'argument n'est pas aussi inexpugnable que la première ". Le Dessein en effet n'est pas, ou n'est plus l'unique manière de relier l'origine du " mécanisme merveilleux " de l'œil avec le fait de la vision. Ce lien alternatif " sur lequel l'attention a été fixé par des spéculations récentes, et dont la réalité ne peut pas être mise en question, bien que son adéquation lorsqu'il s'agit de rendre compte de combinaisons véritablement admirables comme celles présentes dans la Nature, soit encore problématique et le restera probablement longtemps " est le "principe de la survie des plus aptes ${ }^{25}$. La modification graduelle de structures organiques peu perfectionnées par production de variations héritables dont seules celles qui sont favorables seraient retenues pourrait elle aussi rendre raison de la propriété partagée des parties de l'œil. Cette solution, dit Mill, est "prima facie improbable » : elle suffit pourtant à rendre la conclusion de l'argument du Dessein résistible. La théologie naturelle reconnaît, lorsque nous sommes confrontés à une structure complexe adéquate à une fin, qu'il est nécessaire d'avoir recours à un raisonnement abductif pour expliquer d'où vient cette structure : mais la solution qu'elle propose pour que la structure de l'œil cesse d'être surprenante n'est pas la seule qui puisse être envisagée. Mill prend soin de préciser que si on adopte la théorie de l'évolution

\footnotetext{
${ }^{24}$ Mill, X, p. 448.

${ }^{25}$ Ibid., p. 449. Mill retient donc la formule de Spencer (survival of the fittest) pour qualifier le concept darwinien de sélection naturelle. Sur la relation entre survie des plus aptes et sélection naturelle, voir Gayon, 1992, p. 6769.
} 
proposée par Darwin, on n'est pas contraint à abandonner nécessairement l'idée d'une Création (la théorie de l'évolution, comme conception de l'histoire de la vie, demeure neutre sur la question de l'origine de la vie): cependant cette théorie affaiblit considérablement les éléments de preuve en faveur d'une telle Création. Toute la question alors est de savoir si adopter cette théorie de préférence au Dessein est un choix justifié, et justifié au moment où il examine la question.

\section{Mill sur Darwin : forces et faiblesses de la méthode hypothétique}

Dans une lettre du 16 juillet 1861, Henry Fawcett devait s'adresser à Darwin dans ces termes : " je passais une soirée la semaine dernière avec mon ami, M. John Stuart Mill, et je suis certain que vous serez content d'apprendre, venant d'une telle autorité, qu'il considère que votre raisonnement tout du long s'accorde de la manière la plus exacte avec les principes les plus stricts de la logique $»^{26}$. Mill lui-même devait s'exprimer sur l'Origine des espèces dans un passage en note ajouté à la Logique à partir de l'édition de 1862 . Si on regarde de près, ce texte est bien plus nuancé que ce qu'on pouvait attendre à partir de la lettre d'Henry Fawcett, et c'est sur lui que fait fonds la position développée ultérieurement dans l'Essai sur le théisme :

"La spéculation remarquable de Monsieur Darwin est un autre exemple irréprochable d'hypothèse légitime. Ce qu'il nomme " sélection naturelle " n'est pas seulement une vera causa, mais une [vera causa] dont il est prouvé qu'elle est capable de produire des effets du même type que celui que I'hypothèse lui attribue : la question de la possibilité est entièrement une question de degré [...] Il est déraisonnable d'accuser Monsieur Darwin (comme cela a été le cas) de violer les règles de l'Induction. Les règles de l'Induction sont concernées par les conditions de [l'administration de] la Preuve. Monsieur Darwin n'a jamais prétendu que sa doctrine était prouvée. II n'a pas été lié par les règles de I'induction, mais par celles de l'hypothèse. Et ces dernières ont rarement été plus complètement respectées. Il a ouvert un chemin d'investigation plein de promesses, dont nul ne peut prédire les résultats. Et n'est-ce pas un merveilleux exploit en matière de connaissance scientifique et d'ingéniosité que d'avoir rendu une suggestion si hardie, que le premier mouvement de chacun était de

\footnotetext{
${ }^{26}$ Cité par Snyder, 2006, p. 194.
} 
rejeter aussitôt, admissible et discutable, même si c'est au titre de conjecture ? ${ }^{27}$.

Remarquons tout d'abord que Mill insère sa remarque sur Darwin dans un chapitre (III, XIV) où il traite de la méthode hypothétique. De l'hypothèse, Mill propose la définition suivante: "Une hypothèse est quelque supposition que nous faisons (soit sans éléments de preuve véritables, soit avec des éléments de preuve reconnus comme insuffisants) de manière à nous efforcer de déduire à partir d'elle des conclusions qui sont en accord avec des faits connus pour être réels ${ }^{28}$. Comme l'a remarqué John Skorupski, ce n'est pas la fécondité heuristique des hypothèses que Mill met en doute, mais la capacité de la méthode hypothétique à nous conduire à de nouvelles vérités ${ }^{29}$. Pour Mill, la méthode hypothétique est une méthode déductive tronquée, où le chercheur se dispense de la première phase, "l'induction " proprement dite, qui doit établir la « loi de la cause ». C'est l'absence d'induction qui est responsable du fait que l'hypothèse soit présentée, comme on l'a vu, "soit sans éléments de preuve véritables, soit avec des éléments de preuve reconnus comme insuffisants ». En d'autres termes, Mill nie qu'une hypothèse puisse être évaluée uniquement à partir d'une part de sa plausibilité intrinsèque, et d'autre part de ses mérites explicatifs, c'est-à-dire de sa capacité à rendre raison de faits avérés. La justification de cette seconde réserve réside dans l'idée qu'il ne faut jamais exclure la possibilité d'une explication de la même classe de faits qui émanerait d'hypothèses rivales (ce que le lecteur contemporain ne peut pas ne pas rapprocher des préoccupations qui seront celles de Quine ultérieurement lorsqu'il traite de la possibilité de systèmes du monde empiriquement équivalents). Les mérites explicatifs d'une hypothèse ne doivent pas nous conduire à l'adopter de manière précipitée. Il est frappant de ce point de vue, que Mill, lorsqu'il insiste sur ce point, examine longuement l'hypothèse de l'éther ce qu'il fait dans un contexte scientifique où la théorie ondulatoire de la lumière est largement acceptée. Car c'est ce même exemple de l'éther luminifère qui est invoqué par Darwin en 1868 lors de sa défense de sa théorie : "Dans les investigations scientifiques, il est permis d'inventer quelque hypothèse que l'on veut, et si elle explique diverses classes de fait à la fois vastes

\footnotetext{
${ }^{27}$ Mill, VII, p. 498-499. Je retraduis le texte.

${ }^{28}$ Mill, VII, p. 490.

${ }^{29}$ Skorupski, 1989, p. 197.
} 
et indépendantes, elle s'élève au rang de théorie bien fondée. Les ondulations de l'éther sont hypothétiques, et même son existence, cependant chacun admet à présent la nature ondulatoire de la lumière $~_{30}$. Dans le chapitre de la Logique que nous examinons, Mill estime que l'hypothèse de l'éther est loin d'être intégrée à la théorie des phénomènes lumineux d'une manière complètement satisfaisante, et rétrospectivement sa critique paraît perspicace.

Comme on sait, Darwin a une raison particulière de considérer que les mérites explicatifs de sa théorie la mettent à l'abri des critiques : la manière dont son argumentation se conforme dans une large mesure au modèle de ce que Whewell a appelé la consilience des inductions ${ }^{31}$, qui est " le moyen par lequel nous effectuons la généralisation successive qui constitue l'avancement de la science ${ }^{32}$. La consilience se présente sous la forme d'une unification causale, lorsque la cause qui explique les phénomènes de type $A$ (dans l'exemple paradigmatique newtonien, le mouvement des planètes), se trouve également expliquer les phénomènes de type $B$ (la chute des corps), et de type $C$ (le phénomène des marées), c'est-à-dire des classes de phénomènes qu'elle n'était pas destinée à expliquer initialement. La "succession géologique des êtres organiques dans les temps passés et présents ${ }^{33}$ " par exemple, peut être considérée comme une classe de faits dont on peut rendre raison au moyen de la théorie que présente Darwin sans être ce que cette théorie est initialement conçue pour expliquer. Mill ne discute jamais directement la notion de consilience dans la Logique en la nommant, mais lorsqu'il évoque l'idée ${ }^{34} c^{\prime} e s t$ pour affirmer que la tendance est plutôt à en exagérer l'importance comme critère de confirmation: la consilience ne peut se substituer au processus inductif qui transforme l'hypothèse en théorie confirmée. Du fait de l'opposition systématique de la Logique à la philosophie des sciences de Whewell dont la consilience des inductions est un élément de première importance, la manière dont Darwin s'en réclamait dans sa stratégie argumentative ne pouvait pas rencontrer d'écho favorable chez Mill.

\footnotetext{
${ }^{30}$ Darwin 1868, p. 9.

${ }^{31}$ Ruse, 1975, p. 168.

32 Snyder, 2006, p. 175.

${ }^{33}$ Darwin, 1868, p. 9.

${ }^{34}$ Mill, VII, p. 462.
} 
Un autre aspect du jugement de Mill sur Darwin dans la Logique est l'appel à la notion de cause véritable (vera causa). Dans ce même chapitre XIV du Livre III, Mill a reconnu qu'une vraie cause n'est pas nécessairement une cause déjà connue mais que "son existence doit être susceptible d'être connue, et sa connexion avec l'effet qui lui est attribué doit être susceptible d'être prouvée au moyen d'éléments de preuve indépendants. L'hypothèse, en suggérant des observations et des expériences, nous met sur la voie de ces éléments de preuve indépendants, si ceux-ci peuvent être réellement atteints; et jusqu'à ce qu'ils soient atteints, l'hypothèse doit seulement compter comme une conjecture plus ou moins plausible ${ }^{35} "$. Ce texte, outre la référence au différend entre Herschel et Whewell sur ce qui caractérise une cause véritable ${ }^{36}$, permet de percevoir ce qui fonde le jugement positif de Mill sur l'Origine des Espèces, et en même temps ce qui restreint la portée de ce jugement. En affirmant que la sélection naturelle est une vera causa, Mill souligne qu'elle peut être connue et étudiée dans sa connexion avec ses effets au moyen d'éléments de preuve indépendants, qu'elle n'est donc pas une simple hypothèse, à la différence par exemple d'un autre exemple qu'il examine, celui de l'hypothèse des tourbillons de Descartes. A ce sujet, Mill pense vraisemblablement à ce que Darwin appelle en 1868 la "formation analogique des races domestiques " ${ }^{37}$, c'est-à-dire à la sélection des éleveurs. Aux yeux de Mill, rien n'empêche ensuite de développer un programme de recherche qui étudiera l'extension des effets de la sélection et qui permettra d'évaluer la portée de son pouvoir explicatif. L'hypothèse darwinienne est donc justifiée par son contenu empirique, et elle est féconde (plutôt que stérile). Mais selon Mill, comme on ne peut pas savoir à l'avance si ce mode d'explication permettra de pousser l'explication des phénomènes naturels aussi loin qu'on peut le souhaiter, et de valider l'idée d'un pouvoir de la sélection aussi large que celui que Darwin lui attribue, ce n'est pas assez pour donner raison à Darwin. Darwin estime qu'on explique grâce au principe de la sélection de « vastes classes de faits indépendants " ${ }^{38}$, Mill estime qu'on pourrait les expliquer ainsi. Ceci a une conséquence directe sur l'évaluation comparée de l'argument du Dessein et de l'hypothèse darwinienne. Mill ne compare pas la

\footnotetext{
${ }^{35}$ Mill, VII, p. 496.

${ }^{36}$ A propos de ce différend, voir Snyder, 2006, p. 200-202.

${ }^{37}$ Darwin, 1868, p. 9.

38 Ibid.
} 
proposition de Darwin au Dessein comme un programme de recherche fécond à une position dogmatique et stérile. II compare en fait une hypothèse dont l'avenir seul nous dira si elle peut être davantage qu'une conjecture plausible, à un mode d'explication qui possède en lui-même, dès à présent, ses vertus propres. D'où les formules relatives à Darwin dans l'essai « Théisme " : en faveur de cette "théorie ", qu'il appelle aussi une "histoire hypothétique de la nature ", il existe des " analogies qui ont été découvertes dans l'expérience, favorables à sa possibilité ", et " elles excèdent de beaucoup ce que quiconque aurait pu supposer à l'avance ${ }^{39}$. Voilà pour l'éloge. Mais on ne peut pas prédire avec certitude jusqu'où cette théorie se trouvera confirmée et la conclusion de la comparaison entre le Dessein et Darwin, ne peut qu'être une conclusion provisoire, datée, "en l'état de notre connaissance ${ }^{40}$. Cette conclusion est favorable au Dessein, la création par l'intelligence étant bien plus probablement l'explication correcte des adaptations dans la nature.

Darwin se retrouve ainsi dans une position doublement désavantageuse vis-à-vis des principaux philosophes des science de son temps. D'une part, l'Origine des espèces propose une explication de l'ordre naturel divorcée du Dessein qui ne pouvait qu'être rejetée par Whewell, un philosophe dont il avait largement fait sienne, par ailleurs, la conception de la science. D'autre part, par l'assimilation de sa démarche à la méthode hypothétique, étrangère au régime de la preuve, et par le rôle qu'il fait jouer à la consilience des inductions, Darwin est pour Mill quelqu'un qui défend habilement ce qui reste une conjecture et dont la théorie ne satisfait nullement en l'état les normes d'une induction scientifique pleinement probante. Tout se passe ainsi finalement comme si Mill retirait d'une main ce qu'il accorde à Darwin de l'autre, ce qui aboutit dans l'Essai sur le Théisme à une hiérarchie des degrés de probabilité qui permet au Dessein de l'emporter, même si c'est une victoire incomplète.

\section{La métaphysique millienne et la question du naturalisme}

A la question de savoir pourquoi Mill ne se saisit pas de l'Origine des espèces pour ruiner l'argument du Dessein, la réponse ne peut cependant pas être purement épistémologique au sens d'un examen des normes de scientificité telles que la Logique les met en oeuvre. Car l'analyse millienne de la syntaxe des raisonnements met délibérément du côté, comme ne devant pas entrer en

\footnotetext{
${ }^{39}$ Mill, X, p. 449.

${ }^{40}$ Ibid., p. 450.
} 
compte, ce qui peut nous sembler devoir être déterminant, la différence entre le surnaturel et le naturel. Selon Mill, la référence au surnaturel ne disqualifie pas a priori l'argument du Dessein. Pire : elle ne suffit même pas à l'affaiblir. II faut pour le comprendre à la fois préciser sur un point important la caractérisation de la théorie de la connaissance millienne et se tourner vers sa métaphysique. Ce qui transparaît à travers l'examen de l'argument du Dessein, c'est finalement la complexité des relations que la pensée de Mill entretient avec le naturalisme.

Dans la section du même Essai consacrée à l'immortalité de l'âme, Mill traite de la relation entre esprit et cerveau avec une prudence caractéristique. Ce passage mérite d'être mentionné, car la relation entre croyance en l'immortalité d'une âme immatérielle et connaissance du cerveau n'est guère différente de celle qui unit Dessein et biologie. Quand on a montré que "le développement des facultés mentales " s'accompagne des " degrés différents de complication de l'organisation nerveuse et cérébrale ", que " les maladies du cerveau perturbent les fonctions mentales » et que "la sénescence ou la déficience du cerveau les affaiblit ", on possède des éléments de preuve suffisants pour affirmer que "l'action cérébrale est, sinon la cause, du moins dans notre condition d'existence présente, une condition sine qua non des opérations mentales". Mais établir cette proposition ne revient qu'à " un manque de preuve " : ces considérations n'offrent " aucun argument positif contre l'immortalité ${ }^{41}$. Tout d'abord, celui qui croit pouvoir inférer des données des sciences du cerveau que le monisme matérialiste est vrai est quelqu'un qui change sans s'en rendre compte et de manière indue une vérité empirique sur la consécution des phénomènes en une proposition métaphysique sur la nature du monde. II prend sa préférence à priori pour le monisme pour une vérité tirée de l'expérience ; il confond des corrélations de fait avec des vérités de raison, alors que la relation entre esprit et cerveau n'a aucun caractère de nécessité -rien ne nous empêche d'imaginer une situation contrefactuelle où les phénomènes mentaux demeurent et où leurs corrélats cérébraux sont supprimés. Selon Mill, le monisme matérialiste est une variante de philosophie a priori, peu différente du spiritualisme qu'il prétend combattre. II se réclame de la science mais n'est pas justifié par ce que la science établit. De plus, pour Mill, celui qui veut que le monisme matérialiste l'emporte commet une seconde erreur : le cerveau « tout comme les fonctions mentales, est comme la matière elle-même, simplement

\footnotetext{
${ }^{41}$ Mill, X, p. 461.
} 
un ensemble de sensations soit réelles soit inférées comme possibles, c'est-àdire celles qu'a l'anatomiste lorsqu'il ouvre le crâne, ou celles qu'on suppose qu'il recevrait de mouvements moléculaires ou autres lorsque l'action cérébrale se poursuit, dans le cas où il n'y aurait pas d'enveloppe osseuse ou bien où nos instruments seraient suffisamment délicats ${ }^{42}$. Le matérialiste ordinaire croit que le cerveau est une entité substantielle dont la réalité est ultime et dont la science nous révèle la nature. Mais " cerveau " est simplement le nom de régularités dans la succession de nos sensations réelles et possibles. Et on peut ajouter, puisque dans ce même passage, Mill met en parallèle matérialité de la vie et matérialité de la pensée : les organismes sont eux aussi des régularités dans la succession de nos sensations réelles et possibles. Au-delà de l'esprit et de sa présence à lui-même, tout le reste est conjectural.

C'est au chapitre XI d'Un examen de la Philosophie de sir William Hamilton que Mill a précisé sa métaphysique phénoménaliste, dont le motif fondamental est la définition de la matière comme " possibilité permanente de la sensation ${ }^{43}$, et dont le passage ci-dessus de "Théisme " est l'application à des cas particuliers. Le phénoménisme est la variante chez Mill de la thèse de la "relativité de la connaissance humaine " telle qu'il reproche à Hamilton de ne pas l'avoir soutenue avec assez de vigueur et de constance. Mill rejette l'idée d'une connaissance de la nature intrinsèque des composants du monde et sa théorie inductiviste de la connaissance rencontre son phénoménisme en ceci que l'une comme l'autre s'opposent au réalisme scientifique ${ }^{44}$. Dès lors, s'il y a bien un naturalisme de Mill, ce naturalisme est méthodologique : il concerne la validité du modèle de connaissance des sciences de la nature et leur extension possible (et fondée) aux phénomènes mentaux et sociaux qui peuvent être approchés avec les mêmes méthodes et sont susceptibles de recevoir des explications du même type que les phénomènes étudiés par les sciences de la nature. Mais ce naturalisme méthodologique ou "syntaxique " n'implique aucun engagement naturaliste sur le plan métaphysique (où les limites de la nature seraient les limites de ce qui est, et de ce qui est intelligible). C'est sans doute une condition d'arrière-plan de l'examen de l'argument du Dessein comme d'un argument d'une nature "réellement scientifique ${ }^{45}$ ». En s'accordant avec la méthode de

\footnotetext{
${ }^{42}$ Ibid., p. 461-2.

${ }^{43}$ Mill, IX, p. 183.

${ }^{44}$ Skorupski, 1989, chapitre 7, en particulier p. 205.

${ }^{45}$ Mill, X, p. 446.
} 
concordance, l'argument suit les canons de l'induction et le disqualifier parce qu'il implique un agent supranaturel serait statuer a priori sur ce qui peut être. La rationalité scientifique est pour Mill une rationalité procédurale et en définitive, déterminer l'ameublement du monde n'est pas du ressort de la science. Le seul correctif qu'on doive ajouter est que des considérations du type de ce qu'on appelle depuis Rawls l'équilibre réfléchi ne sont pas étrangères à Mill : selon lui le principe d'uniformité du cours de la nature et la découverte de lois invariantes dans des domaines particuliers se confortent mutuellement ${ }^{46}$, et de même l'attrait du Dessein et des hypothèses métaphysiques qu'il implique ne peuvent que diminuer considérablement si la voie ouverte par Darwin est la source de généralisations robustes.

L'espoir qu'on s'accorde (The indulgence of hope)

Lorsque Mill conclut l'Essai sur le Théisme avec une partie qui en expose le "Résultat général », il soutient que l'attitude rationnelle de l'esprit vis-à-vis du surnaturel est le scepticisme. L'attitude sceptique se distingue à la fois de la croyance, et de l'athéisme ${ }^{47}$. Cependant, comme l'athéisme a deux formes, le déni dogmatique de l'existence de Dieu, mais aussi « le fait de nier qu'il y ait des éléments de preuve dans un sens ou dans l'autre, ce qui pour la plupart des buts pratiques revient à avoir prouvé que Dieu n'existe pas ", le scepticisme que Mill préconise ne consiste pas à se tenir également éloigné de la croyance et de l'athéisme. L'athéisme a tort en effet lorsqu'il estime que rien ne peut être dit en faveur de l'existence d'une forme de divinité, quand bien même il a entièrement raison de rejeter la croyance en un Dieu omnipotent et en un gouvernement du monde par la providence. Renoncer à la croyance au surnaturel n'est pas donner raison à l'athéisme et le scepticisme autorise en fait, selon la formule de Mill, de « retirer le vaste domaine du surnaturel de la région de la Croyance pour le transférer dans celle du simple Espoir ${ }^{48}$. L'imagination, comme le disait déjà l'Essai "Utilité de la religion ", peut être peuplée de possibilités aussi longtemps qu'elle ne nous conduit pas à l'erreur. Aussi la valeur de l'imagination doit être reconnue, et son usage doit être cultivé.

\footnotetext{
${ }^{46}$ Mill, IX, p. 482, note 1.

${ }^{47}$ Mill, X, p. 482.

48 Ibid., X, p. 483.
} 
Linda C. Reader a interprété l'analyse millienne de l'espoir comme une analyse intéressée : tout à son objectif du remplacement des religions révélées par une religion sans Dieu qui aurait l'Humanité comme objet et le progrès moral comme objectif ; convaincu, comme il l'avait écrit dans son livre sur Auguste Comte et le positivisme, que les religions ordinaires sont d'autant plus perfectionnées que leurs effets pratiques coïncident avec celles de la religion de l'Humanitét9, Mill concèderait l'espoir pour obtenir de ses semblables ce qui peut être motivé par l'espoir : " ce que Mill suggère est que l'espoir en un Dieu et en l'immortalité, entretenu par l'imagination, peut servir à engendrer une émotion et une motivation suffisants pour rallier les masses à sa nouvelle Religion de I'humanité ${ }^{50}$. L'interprétation proposée par Reader est suggestive, mais elle demande à être corrigée. Rien ne serait moins millien, en effet, que l'idée d'une instrumentalisation de la crédulité humaine qui permettrait de façonner les conduites en feignant d'approuver les croyances qui sont à l'arrière-plan de celles-ci.

Tout d'abord, s'il s'agit bien de conjoindre une religion tournée vers un Dieu et une religion purement humaine, il faut encore définir quel type de religion admettant une forme de transcendance peut entrer dans une telle conjonction. Dans l'Essai « Utilité de la religion », Mill avait ainsi fait l'éloge du Manichéisme ${ }^{51}$, Manichéisme dont il rappelait dans son Autobiographie que son père James Mill ne le rejetait pas comme il rejetait d'autres formes de croyance religieuse ${ }^{52}$. Le Manichéisme est cette position où à la fois, la pensée s'épargne les contorsions du providentialisme et où tout agent qui tend à faire advenir le bien dans le monde travaille aux côtés d'un Dieu bon avec lequel il coopère : l'agent moral se représente alors comme un " compagnon de lutte » de la Divinité, engagé dans un combat dont l'issue est le sort du monde. D'une part, chaque décision dans le sens du bien commun est alors confortée par l'action dans le même sens de la divinité, et d'autre part, puisque la divinité bonne n'est pas représentée comme dotée d'un pouvoir infini, la responsabilité du sujet moral et le poids de ses décisions s'en trouvent renforcées : chacun en agissant dans un sens ou dans un

\footnotetext{
${ }^{49}$ Ibid., X, p. 334-335 et traduction, p. 146.

${ }^{50}$ Reader, 2002, p. 225.

${ }^{51}$ Mill, X, p. 425.

52 Ibid., I, p. 43 et Reader, 2002, p. 10-11, 138-139.
} 
autre, peut là où il intervient faire la différence, si minime soit-elle. Dans "Théisme ", Mill analyse cette fois la figure du Christ comme celle d'un idéal moral qui appelle l'imitation et dont l'approbation est recherchée. La religion telle que la perçoit Mill permet à l'agent moral de se représenter une communauté morale à laquelle, si isolé qu'il soit, il appartient à chaque instant du fait de son propre vouloir. Dès lors, se permettre l'espoir est se conforter dans sa capacité à agir dans le meilleur sens. La religion de l'humanité ne dépend pas dans sa possibilité de l'espérance donnée par l'imagination d'un Dieu visant le bien auquel nous viendrions prêter notre concours, mais notre capacité subjective à œuvrer résolument dans le sens de cette religion de l'humanité peut se trouver renforcée par l'espoir religieux. Cette possibilité est donnée à ceux qui en ont " besoin ", et rien ne dit dans le texte qui aura plus particulièrement besoin de l'espoir. On peut penser que le contenu même de l'espoir peut varier ; ainsi l'espoir en la vie future est selon Mill (c'est la fin de l'Essai « Utilité de la religion ${ }^{53}$ ) un espoir dont nous pouvons expliquer l'importance que les hommes lui accordent en invoquant une évolution sociale inachevée où l'appétit d'une vie éternelle est augmenté par la misère morale et matérielle de l'existence présente, ce qui implique qu'on peut prédire qu'il diminuera à mesure que la condition humaine sera améliorée. Mais comme on l'a vu, il ne s'agit pas pour Mill de ce que la religion promet de plus important, ce qui compte est l'imagination qui permet à l'agent moral d'échapper à la solitude.

En second lieu, l'espoir serait déraisonnable si l'examen rationnel avait abouti à une condamnation du théisme. Or on a vu qu'il n'en est rien, et on ne peut pas soupçonner Mill d'avoir infléchi l'analyse du Dessein pour aller dans le sens de l'espoir. Dans l'équilibre délicat de la philosophie millienne de la religion que l'on trouve dans cette partie conclusive du Troisième Essai, le jugement porté sur l'argument du Dessein joue manifestement un rôle décisif. Car en l'absence d'autres éléments probants, c'est le fait qu'on ne puisse invoquer en faveur de l'existence d'un concepteur du monde naturel qu'un élément de preuve (evidence) qui n'a pas en lui-même la valeur d'une preuve complète (proof) et possède seulement "l'un des degrés les plus faibles de probabilité ${ }^{54}$, qui à la fois rend le scepticisme préférable à la croyance et à l'athéisme, et en même temps permet d'assumer ce scepticisme en l'infléchissant rationnellement, tout bien pesé, dans le sens de l'espoir. Le Dieu moral du Manichéisme aux pouvoirs

\footnotetext{
53 Ibid., p. 426-28.

${ }^{54}$ Ibid., X, p. 482.
} 
limités tel qu'on le rencontre dans "Utilité de la religion " coïncide alors avec le Dieu du Dessein qui dans "Théisme " n'est pas responsable de l'existence du monde mais pourrait bien avoir façonné l'ordre naturel. Affaiblir l'argument du Dessein au point de devoir renoncer entièrement, non seulement à la croyance, mais à la pensée de son objet, ce serait perturber l'équilibre fragile auquel Mill parvient entre le rejet du dogme religieux et le rôle motivationnel de l'espoir, en tant que celui-ci est placé au service de l'utilitarisme moral. Il faut cependant ajouter que le scepticisme implique également un rejet de la croyance, et le fait que Darwin ait proposé selon la formule de la Logique un " exemple irréprochable d'hypothèse légitime ${ }^{55}$ vient contribuer à empêcher que les éléments de preuve en faveur du Dessein conduisent à tort à une certitude complète et à une restauration de la croyance.

En d'autres termes, on ne peut blâmer un espoir raisonnable qui conduit à une attitude devant l'existence elle-même irréprochable, et on peut encore moins le blâmer s'il est présentement la source d'une motivation qui n'a pas d'équivalent et dont l'agent peut avoir besoin pour faire le bien. On ne peut manquer de remarquer comment, chez cet auteur anti-kantien entre tous qu'est Mill, la survivance de la religion dans l'espoir vient finalement faire le lien, même si c'est un lien fragile, entre le domaine de la connaissance et celui de la moralité.

\section{Conclusion}

Au début de "Théisme", Mill fait une distinction entre deux traitements possibles de la religion, I'un « historique ", l'autre " dogmatique ». Le traitement historique est celui qui examine la religion, non pas en termes de vérité et de fausseté, mais comme le produit d'un état de civilisation: plutôt que de dénoncer la religion comme erreur, il conduit à s'intéresser aux services qu'elle a pu rendre, ou qu'elle rend encore, dans un certain état social ${ }^{56}$. Le point de vue " dogmatique ", lui, s'intéresse à la vérité et à la fausseté des croyances, et à l'examen des éléments de confirmation. La religion doit alors être alors évaluée selon les normes qui sont celles de la science. Si l'examen de l'argument du Dessein par Mill relève de cette seconde approche, il a pour contexte une

\footnotetext{
${ }^{55}$ Ibid, VII, p. 498-99.

${ }^{56}$ Dans l'affirmation de Durkheim selon laquelle les religions sont « toutes vraies à leur façon ", le point de vue historique sur les mérites passés de la religion se transforme en une affirmation sur son rôle dans chacune des sociétés où nous la rencontrons.
} 
interrogation sur l'utilité de la religion qui en éclaire la place dans l'économie de la pensée de Mill. Avec l'alliance du scepticisme et d'une imagination qu'il n'est ni nécessaire, ni bénéfique de brider, Mill prend acte de l'affaiblissement des fondements rationnels de la croyance, tout montrant comment le progrès du monde moral peut bénéficier d'une forme d'espérance religieuse qui élargit et fortifie la communauté des agents du progrès. La philosophie de la religion de Mill est alors le point de convergence entre sa métaphysique, sa théorie de la connaissance et sa philosophie morale, soit entre son phénoménalisme, son inductivisme et son utilitarisme. Le Dessein ne prouve pas Dieu, mais il suffit à justifier l'espoir, un espoir moins tourné vers la vie future de l'individu que vers l'amélioration du monde partagé.

Au sujet de la relation entre Mill et Darwin, on pourrait être tenté de parler d'une rencontre manquée, qui mérite d'autant plus l'analyse que le rejet résolu par Mill d'un gouvernement moral de l'Univers par un Dieu omnipotent a une parenté évidente avec le rejet par Darwin d'une téléologie " trivialisée " (comme disait David Hull) où Dieu aurait voulu chaque variation et serait derrière toutes les modifications et tous les accidents du monde naturel ${ }^{57}$. Pour l'un, comme pour l'autre, "tout ce qui est, a été voulu » est une proposition vide de sens. $S^{\prime}$ interroger sur la réserve de Mill vis-à-vis de Darwin impose de ne pas oublier quel en est l'objet, et quel en est l'arrière-plan. D'une part, un siècle et demi d'évolution de la théorie de l'évolution nous sépare du Darwin que Mill examine dans ses écrits: nous donnons d'autant plus tort à Mill que nous augmentons Darwin d'une postérité que l'auteur de la Logique ne peut connaître. D'autre part, lorsque Mill se refuse à donner raison à Darwin, il se refuse en fait à donner raison à la science parce qu'il estime qu'en ce cas la science ne peut faire comme si elle avait accompli son programme, parce qu'il estime ne pas pouvoir ignorer qu'elle n'a pas réuni toutes les raisons qui permettraient de lui donner raison. Le choix du Dessein contre Darwin peut nous paraître erroné, quand bien même il est présenté par Mill d'une manière telle qu'il est ouvert à la révision. Il faut le lire cependant selon une exigence vis-à-vis de la science qui ne procède d'aucune hostilité à son égard. Mill nous rappelle que la confiance en la science ne peut être inconditionnelle sans trahir son intention. Si nous pouvons combattre le

\footnotetext{
${ }^{57}$ Lettre de Darwin à Lyell, 13 août 1861 (Hull, 1973, p. 63) : « si vous dites que Dieu a ordonné qu'en un certain temps et un certain lieu une douzaine de petites variations se produisent, et que l'une d'elles soit préservée dans la lutte pour la vie et que les onze autres périssent à la première ou après quelques générations, cela me paraît être un pur verbiage. Cela revient simplement à dire que tout ce qui est, est, est le produit d'une volonté (ordained) ".
} 
rejet par Mill du réalisme scientifique qu'impose son phénoménisme, si nous pouvons estimer que le souci millien de préserver le libre examen des hypothèses le retient à tort de présenter l'apport de Darwin sous un jour plus favorable et plus équitable, le souci qui est le sien d'interroger les propositions particulières de la science à partir des normes de la scientificité conserve toute sa valeur.

\section{Denis Forest}

\section{BIBLIOGRAPHIE}

DARWIN Charles, The Variation of animals and plants under domestication, Volume 1, première publication 1868, Baltimore et Londres, The Johns Hopkins University Press, 1998.

GAYON Jean, Darwin et l'après-Darwin : une histoire de I'hypothèse de sélection naturelle. Paris, Kimé, 1992.

HULL David, Darwin and his critics, Harvard University Press, 1973.

HUME David, Dialogues sur la religion naturelle. Texte, traduction et commentaire de Michel Malherbe, Paris, Vrin, 2005.

MERLEAU-PONTY Jacques, La science de l'univers à l'âge du positivisme, Paris, Vrin 1983.

MILL John Stuart, Autobiography, in The Collected works of John Stuart Mill, 33 vol. Toronto, University of Toronto Press, Volume I.

A system of Logic, in The Collected works, Volumes VII et VIII.

volume IX.

An examination of the philosophy of sir William Hamilton, in The Collected works,

Auguste Comte and positivism in The Collected works, volume X. Traduction Georges Clémenceau, Paris, L’Harmattan, 1999, texte revu et présenté par Michel Bourdeau.

Three Essays on Religion, in The Collected Works, volume X.

RUSE Michael, "Darwin's debt to Philosophy”, Studies in the History and Philosophy of Science, 6 (1975), p. 159-181.

READER Lynda C. John Stuart Mill and the religion of humanity, University of Missouri Press, Columbia et Londres, 2002.

SKORUPSKI John, John Stuart Mill, Routledge, 1989.

SNYDER Laura, Reforming Philosophy, University of Chicago Press, 2006.

VAN FRAASSEN Bas C., The empirical stance, Yale University Press, 2002. 
WHEWELL William, Astronomy and general physics, considered with Reference to Natural Theology, Bridgewater Treatise, III, Londres, William Pickering, 1833; Nouvelle édition, 1864, réédition Thoemmes Press, 2001.

Indications of the creator. Extracts, bearing upon Theology, from the History and the Philosophy of the Inductive sciences, seconde édition, Londres, John W. Parker, 1846.

The History of the inductive sciences, Londres, John W. Parker, 1857, réédition Thoemmes Press, 2001.

The Philosophy of the inductive sciences, founded upon their history, Londres, John W. Parker, 1847, réédition Thoemmes Press. 